KRAUSE, MR; Lo MONACO, PAV; HADDADE, IR; MENEGHELLI, LAM; SOUZA, TD. 2017. Aproveitamento de resíduos agrícolas na composição de substratos para produção de mudas de tomateiro. Horticultura Brasileira, 35:305-310. DOI - http://dx.doi.org/10.1590/S0102-053620170224

\title{
Aproveitamento de resíduos agrícolas na composição de substratos para produção de mudas de tomateiro
}

\author{
Marcelo R Krause ${ }^{1}$; Paola AVL Monaco ${ }^{1}$; Ismail R Haddade'; Lorena AM Meneghelli ${ }^{1}$; Tamara D Souza ${ }^{2}$ \\ ${ }^{1}$ Instituto Federal do Espírito Santo (IFES), Santa Teresa-ES, Brasil; agro.krause@gmail.com; paolalm@ifes.edu.br; ihaddade@gmail. \\ com; lorena.merlo@hotmail.com; ${ }^{2}$ Universidade Federal de Viçosa (UFV), Viçosa-MG, Brasil; tamaradaianesouza@gmail.com
}

\section{RESUMO}

Estudos relacionados ao aproveitamento de resíduos agrícolas tornaram-se imprescindíveis pela possibilidade de seu uso na produção de mudas de olerícolas e por minimizar o risco de contaminação ambiental decorrente do descarte inadequado. O objetivo do trabalho foi avaliar o crescimento de mudas de tomateiro produzidas em substratos compostos por fibra de coco, casca de ovo e com níveis crescentes de moinha em substituição ao substrato comercial Bioplant. As composições dos substratos testados foram: $\mathrm{T} 0=$ substrato comercial; $\mathrm{T} 1=15 \%$ de moinha (MO: resíduo da secagem dos grãos de café) $+15 \%$ fibra de coco $(\mathrm{FC})+5 \%$ casca de ovo $(\mathrm{CO})+65 \%$ substrato comercial (SC); $\mathrm{T} 2=30 \% \mathrm{MO}+15 \% \mathrm{FC}+5 \% \mathrm{CO}+50 \%$ $\mathrm{SC} ; \mathrm{T} 3=45 \% \mathrm{MO}+15 \% \mathrm{FC}+5 \% \mathrm{CO}+35 \% \mathrm{SC}$; $\mathrm{T} 4=60 \% \mathrm{MO}+$ $15 \% \mathrm{FC}+5 \% \mathrm{CO}+20 \% \mathrm{SC}$. Número de folhas, altura de plântula, diâmetro de caule, massa fresca e seca da parte aérea, de raiz e da planta inteira foram avaliados. Os substratos alternativos contendo de 15 a $32 \%$ de moinha $+15 \%$ fibra de coco $+5 \%$ casca de ovo + $48 \%$ a $65 \%$ de substrato comercial proporcionam os maiores valores de todas as variáveis analisadas. Todas as proporções de moinha estudadas promovem valores das variáveis de crescimento superiores àqueles obtidos quando se usa somente o substrato comercial. O uso de $5 \%$ casca de ovo, $15 \%$ de fibra de coco, 15 a $32 \%$ de moinha e 48 a $65 \%$ de substrato comercial é uma alternativa de substrato para produção de mudas de tomate, permitindo um adequado destino a esses resíduos agrícolas.

Palavras-chave: Solanum lycopersicum, substrato alternativo, moinha, fibra de coco.

\begin{abstract}
Agricultural wastes used as alternative substrates for the production of tomato seedlings

Studies concerning the use of agricultural wastes are essential considering they can be employed on seedling production, minimizing the risk of environmental contamination due to inappropriate disposal. We evaluated growth variables of tomato seedlings produced on substrates composed by coconut fiber, eggshell and increasing levels of moinha replacing the commercial substrate Bioplant. The experiment was carried out in a completely randomized design, with five treatments and ten replications, treatments being: $\mathrm{T} 0=$ commercial substrate; $\mathrm{T} 1=15 \%$ moinh (MO: wastes from the coffee seeds drying) $+15 \%$ coconut fiber $(\mathrm{FC})+5 \%$ eggshell $(\mathrm{CO})+65 \%$ commercial substrate (SC); $\mathrm{T} 2=30 \% \mathrm{MO}+15 \% \mathrm{FC}+5 \% \mathrm{CO}+$ $50 \% \mathrm{SC} ; \mathrm{T} 3=45 \% \mathrm{MO}+15 \% \mathrm{FC}+5 \% \mathrm{CO}+35 \% \mathrm{SC} ; \mathrm{T} 4=60 \%$ $\mathrm{MO}+15 \% \mathrm{FC}+5 \% \mathrm{CO}+20 \% \mathrm{SC}$. Number of leaves; seedling height, stem diameter; fresh and dry matter of root, aboveground part and of the whole plant were evaluated. The alternative substrate containing 15 to $32 \%$ moinha $+15 \%$ coconut fiber $+5 \%$ eggshell + 48 to $65 \%$ commercial substrate provided the highest values for all analyzed variables. All the proportions of moinha in the substrate promoted growth variables values higher than those obtained when using only the commercial substrate. The use of 5\% eggshell, $15 \%$ coconut fiber, 15 to $32 \%$ moinha and 48 to $65 \%$ commercial substrate is an alternative substrate for the production of tomato seedlings, this being a suitable destination for the agricultural wastes.
\end{abstract}

Keywords: Solanum lycopersicum, alternative substrate, moinha, coconut fiber.

(Recebido para publicação em 24 de maio de 2016; aceito em 17 de abril de 2017)

(Received on May 24, 20016; accepted on April 17, 2017)

\begin{abstract}
A produção de mudas de hortaliças, sobretudo da cultura do tomate (Solanum lycopersicum), constitui-se em uma das etapas mais importantes do sistema produtivo, influenciando no desenvolvimento das plantas. Estima-se que $60 \%$ do sucesso de uma cultura está na implantação com mudas de qualidade (Zaccheo et al., 2013).

Um dos fatores relacionados à qualidade das mudas é o tipo de subs-
\end{abstract}

trato utilizado. A obtenção de mudas de qualidade necessita de substratos com boas características físicas, químicas, biológicas e sanitárias (Mesquita et al., 2012). Mais do que exercer a função de suporte às plantas, o substrato deve proporcionar adequado suprimento de ar e água ao sistema radicular (Silva Júnior et al., 2014). Adicionalmente, este deve ser de fácil manejo, baixo custo, alta disponibilidade e ter longa durabilidade.
Os substratos comerciais são amplamente utilizados para a produção de mudas de tomate, constituindo-se em um custo a mais na etapa de produção de mudas. Nesse sentido, a escolha de um substrato para produção de mudas de olerícolas deve ser também baseada em dois critérios essenciais: o custo de aquisição e a disponibilidade do material para produção do substrato (Steffen et al., 2010). 
Considerando a necessidade de reduzir custos com os substratos comerciais e aproveitar a disponibilidade de resíduos agrícolas gerados em cada região, várias pesquisas têm sido realizadas avaliando combinações de substratos comerciais e alternativos na produção de mudas de tomate (Ferreira et al., 2014; Costa et al., 2015; Cerqueira et al., 2015). Além disso, o aproveitamento de resíduos gerados numa determinada região é também uma forma de minimizar os impactos ambientais causados pelo descarte incorreto desses resíduos no ambiente.

Dentre alguns resíduos gerados em elevada quantidade na região serrana do Estado do Espírito Santo destacam-se a fibra de coco, a casca de ovo e o resíduo da secagem dos grãos de café, também conhecido por "moinha".

A fibra de coco tem sido utilizada com sucesso na composição de substratos para produção de mudas, em virtude de sua disponibilidade, seu baixo custo e das suas adequadas propriedades físicas e químicas (Costa et al., 2007; Barreto \& Testezlaf, 2014; Dias et al., 2015; Caldeira et al., 2014). Já a casca de ovo pode contribuir com a qualidade nutricional do substrato, pelo fato de possuir o carbonato de cálcio como principal constituinte em sua composição.

A moinha é composta por restos vegetais tais como folhas, galhos, restos de inflorescências e grãos mal formados do próprio cafeeiro, que, quando secados juntamente com o mesmo, sofrem queima e são liberados do secador. A moinha possui um elevado conteúdo de matéria orgânica, fósforo, potássio e, especialmente, de nitrogênio (Meneghelli et al., 2016). No entanto, relata-se que concentrações maiores que $10 \%$ de moinha no substrato prejudicam o crescimento de mudas de café Conilon, em virtude da elevada condutividade elétrica deste resíduo.

Assim, acredita-se que a substituição parcial dos substratos comerciais, pela combinação de diferentes resíduos agrícolas, possa ser uma alternativa sustentável para a produção de mudas de tomate, uma vez que todos eles apresentam características favoráveis à germinação e ao desenvolvimento das mudas, além de reduzir custos com substratos comer- ciais. No entanto, torna-se necessário saber qual a proporção de moinha e de substrato comercial na composição do substrato alternativo contendo fibra de coco e casca de ovo, que proporcionará a melhor qualidade de mudas de tomate para o plantio.

Face ao exposto, o objetivo do trabalho foi avaliar o crescimento de mudas de tomateiro produzidas em substratos compostos por fibra de coco, casca de ovo e com níveis crescentes de moinha em substituição ao substrato comercial Bioplant.

\section{MATERIAL E MÉTODOS}

O experimento foi conduzido no período de 4 de setembro a 6 de outubro de 2015 no viveiro de produção de mudas do IFES, Campus Santa Teresa, município de Santa Teresa-ES (1848'19's; 4040'32'O; altitude $130 \mathrm{~m}$ ). O clima, segundo a classificação de Köppen, enquadra-se no tipo Cwa (subtropical de inverno seco), com temperatura média anual de $24,6^{\circ} \mathrm{C}$ e precipitação média anual variando entre 700 e $1200 \mathrm{~mm}$. A temperatura e a umidade relativa do ar, durante o período experimental, foram medidas em estação meteorológica instalada nas adjacências do viveiro, variando entre 19,9 a $38,2^{\circ} \mathrm{C}$ e 47,5 a $69,5 \%$, respectivamente. $\mathrm{O}$ viveiro onde foi desenvolvido o trabalho era coberto com tela de polipropileno, o que proporcionava a redução da radiação solar em $50 \%$.

$\mathrm{O}$ delineamento experimental adotado foi inteiramente casualizado, com cinco tratamentos e dez repetições. Cada unidade experimental consistiu de 20 (vinte) mudas, dispostas em duas linhas verticais da bandeja, onde cada célula apresentava um volume de $18 \mathrm{~cm}^{3}$. Foram consideradas úteis onze plantas para cada unidade experimental, desconsiderando nove mudas posicionadas nas laterais da bandeja.

Assim, foram avaliados os tratamentos seguintes: $\mathrm{T} 0=$ substrato comercial (testemunha); $\mathrm{T} 1=15 \%$ de moinha + $15 \%$ fibra de coco $+5 \%$ casca de ovo + $65 \%$ substrato comercial; $\mathrm{T} 2=30 \%$ de moinha $+15 \%$ fibra de coco $+5 \%$ casca de ovo $+50 \%$ substrato comercial; $\mathrm{T} 3=$ $45 \%$ de moinha $+15 \%$ fibra de coco
$+5 \%$ casca de ovo $+35 \%$ substrato comercial; $\mathrm{T} 4=60 \%$ de moinha $+15 \%$ fibra de coco $+5 \%$ casca de ovo $+20 \%$ substrato comercial. A quantidade de resíduos que foi adicionada aos tratamentos foi calculada com base no volume.

Os resíduos utilizados na formulação do substrato alternativo para produção de mudas de tomate foram provenientes da secagem dos grãos de café, denominado como "moinha", a casca de ovo e a fibra de coco. A casca de ovo foi triturada em almofariz, até se obter um pó. A fibra de coco foi seca ao ar durante três dias e, processada em desintegrador, seguida de peneiramento até se obter uma granulometria na faixa de um a dez milímetros. Já a moinha foi utilizada tal como foi coletada, ou seja, sem qualquer tratamento de trituramento ou peneiramento.

A caracterização química dos resíduos utilizados na composição dos substratos para a produção de mudas de tomateiro foi realizada no Laboratório de Solos e Resíduos Sólidos do Departamento de Engenharia Agrícola da Universidade Federal de Viçosa (Tabela 1). A análise química consistiu na determinação da condutividade elétrica $(\mathrm{CE})$, por meio de um condutivímetro de bancada, do $\mathrm{pH}$, por meio de um peagâmetro de bancada, e na quantificação das concentrações de carbono orgânico facilmente oxidável $\left(\mathrm{CO}_{\mathrm{fo}}\right)$, carbono orgânico total $\left(\mathrm{CO}_{\mathrm{T}}\right)$, matéria orgânica (MO), nitrogênio total $\left(\mathrm{N}_{\mathrm{T}}\right)$, fósforo $(\mathrm{P})$ e potássio $(\mathrm{K})$, seguindo-se metodologia descrita por Matos (2015). O pH e a condutividade elétrica foram determinados na solução do substrato obtida por meio de extrator aquoso, utilizando-se como extrator a água deionizada na proporção de 1:2,5. A suspensão foi misturada vigorosamente e as leituras realizadas após repouso de $30 \mathrm{~min}$.

As sementes de tomate, cultivar Santa Clara, foram semeadas em bandejas de poliestireno expandido com 200 células, colocando-se duas sementes por célula. Onze dias após a semeadura (DAS) realizou-se um desbaste deixando apenas uma plântula, a mais vigorosa.

Utilizou-se o sistema de produção de mudas em bandejas suspensas, alocadas em bancadas de alvenaria, irrigadas manualmente duas vezes ao dia, pela 
manhã e à tarde. Não foi realizada adubação com fertilizantes durante o experimento. As avaliações ocorreram aos 24 DAS consistindo na contagem do número de folhas; medição da altura de plântula, diâmetro de caule; pesagem das massas fresca e seca da parte aérea, da raiz e da planta inteira. Para a obtenção da altura da parte aérea foi utilizada régua milimetrada, medindo-se da base do colo até o ápice da planta. $\mathrm{O}$ diâmetro do caule foi obtido por meio de um paquímetro digital com precisão de $0,01 \mathrm{~mm}$. A massa seca da parte aérea foi obtida por meio do corte das mudas na base do colo. Para a obtenção da massa seca radicular, as raízes foram cuidadosamente lavadas em água corrente, sobre peneira. Posteriormente, os materiais foram acondicionados em sacos de papel e colocados em estufa com circulação de ar forçado a $65^{\circ} \mathrm{C}$ por 72 horas, quando atingiram massa constante. Em seguida, foi realizada a pesagem dos materiais em balança eletrônica de precisão de $0,01 \mathrm{~g}$.

Todas as variáveis avaliadas foram submetidas aos testes de normalidade (Lilliefors) e de homeocedasticidade (Barttlet), pressupostos para a validação da análise de variância. Apenas a variável de massa seca radicular não atendeu a estes pressupostos, sendo transformada em logaritmo neperiano (Ln). Assim, nova verificação foi realizada, verificando-se o atendimento desses pressupostos.

Dada a diferença qualitativa entre o tratamento de substrato comercial (T0) e os demais (T1 a T4), pela ausência da fibra de coco e da casca de ovo no primeiro, foi realizada a análise de contraste entre o T0 e os demais. Para as comparações entre os tratamentos T1, T2, T3 e T4 relacionadas ao nível de moinha (tratamentos quantitativos), adotaram-se como procedimento, a decomposição dos graus de liberdade dos tratamentos em modelos de regressão pelo método dos polinômios ortogonais. Para todos os procedimentos foi adotado um erro tipo I igual a 0,05 . Todas as análises estatísticas foram realizadas pelo programa SAEG 9.1 (SAEG, 2007).

\section{RESULTADOS E DISCUSSÃO}

Na Tabela 2 são apresentados os coeficientes, os níveis de significância e os estimadores dos contrastes entre o tratamento de substrato comercial, e aqueles com os diferentes níveis de inserção da moinha no substrato. Observa-se que o grupo de tratamentos onde a moinha esteve presente foi superior ao daquele com uso exclusivo do substrato comercial, isto para todas as variáveis avaliadas. Acredita-se que tal resultado possa estar relacionado à maior concentração de alguns nutrientes presentes na moinha, sobretudo o nitrogênio (Tabela 1), quando comparado ao substrato comercial. De acordo com Maçãs (2008), o N é um dos nutrientes que mais limita o desenvolvimento, a produtividade e a biomassa da maioria das culturas. O nitrogênio é considerado um dos fatores mais relevantes para o aumento da produção, por influenciar a taxa de emergência e expansão da área foliar (Taiz \& Zeiger, 2013).

Ressalta-se que a utilização da casca de ovo e da fibra de coco nas proporções utilizadas neste trabalho também pode ter contribuído positivamente para desenvolvimento das mudas. A casca de ovo, embora tenha menor conteúdo de matéria orgânica entre os resíduos utilizados, contribui com nutrientes, especialmente com o cálcio (Tabela 1), quando se compara com a quantidade existente no substrato comercial (Paixão et al., 2012). Já a fibra de coco, pode ter contribuído não somente com a qualidade química do substrato, em razão do conteúdo de matéria orgânica (Tabela 1), como também com a qualidade física do substrato, proporcionando adequada aeração e retenção de água.

Nas Figuras 1A, 1B, 1C, 1D, 1E, $1 \mathrm{~F}, 1 \mathrm{G}$ e $1 \mathrm{H}$ apresentam-se a altura de plantas (AP), o diâmetro de caule (DC), o número de folhas (NF), a matéria fresca e seca de raiz (MFR e MSR, respectivamente), a matéria fresca e seca de parte aérea (MFPA e MSPA) e a matéria seca total (MST), respectivamente, em função de porcentagens crescentes de moinha na composição dos substratos. As variáveis AP, DC, NF e MFPA apresentaram um comportamento polinomial quadrático, enquanto que as variáveis MFR, MSPA, MSR e MST manifestaram comportamento linear, em função das diferentes porcentagens de moinha na composição dos substratos para as mudas do tomateiro.

A porcentagem estimada de 17,34\% de moinha na composição dos substratos proporcionou maior altura de planta $(15,83 \mathrm{~cm})$, dentre os tratamentos aplicados (Figura 1A). Os menores valores de altura média foram obtidos nos substratos contendo $60 \%$ de moinha $(10,00$ $\mathrm{cm})$. Mesmo assim, tais valores foram superiores aos alcançados por Rodrigues et al. (2010) que obtiveram alturas de mudas de tomate da mesma cultivar, aos 28 DAS, variando entre 4,07 e 4,36 $\mathrm{cm}$, em substratos com porcentagens crescentes de composto orgânico, e por Costa et al. (2007), que obtiveram a maior altura de planta $(6,81 \mathrm{~cm}) \mathrm{com}$ o uso de $100 \%$ de resíduo de algodão como substrato na produção de mudas de tomate da cultivar Kada Gigante,

Tabela 1. Atributos químicos da casca de ovo (CO), fibra de coco (FC) e moinha \{resíduo da secagem dos grãos de café (MO)\} e substrato comercial (SC) utilizados no experimento \{chemical properties of eggshell (CO), coconut fiber (FC), "moinha" (MO) and commercial substrate (SC) used in the research\}. Santa Teresa, IFES, 2015.

\begin{tabular}{|c|c|c|c|c|c|c|c|c|c|}
\hline \multirow{2}{*}{ Resíduos } & \multirow{2}{*}{ pH } & \multirow{2}{*}{$\begin{array}{c}C E \\
(d S / m)\end{array}$} & $\mathrm{CO}_{\mathrm{fo}_{\mathrm{O}}}$ & $\mathrm{CO}_{\mathrm{T}}$ & MO & $\mathbf{N}_{\mathrm{T}}$ & $\mathbf{P}$ & $\mathbf{K}$ & $\mathrm{Ca}$ \\
\hline & & & \multicolumn{7}{|c|}{ (g/kg) } \\
\hline $\mathrm{CO}$ & 9,37 & 0,37 & 28 & 36 & 62 & 8,7 & 0,84 & 0,6 & 319 \\
\hline $\mathrm{FC}$ & 7,15 & 0,09 & 571 & 741 & 1277 & 6,6 & 0,53 & 1,4 & $* *$ \\
\hline MO & 5,60 & 6,49 & 453 & 589 & 1015 & 37 & 1,40 & 7,1 & 23,3 \\
\hline $\mathrm{SC}^{1}$ & 5,62 & - & - & 210 & 522,1 & 6,2 & 15,50 & 4,4 & 18,4 \\
\hline
\end{tabular}

$\mathrm{CE}=$ condutividade elétrica (electrical conductivity); $\mathrm{CO}_{\mathrm{fo}}=$ carbono orgânico facilmente oxidável (easily-oxyded organic carbon); $\mathrm{CO}_{\mathrm{t}}=$ carbono orgânico total (total organic carbon); $\mathrm{MO}=$ matéria orgânica (organic matter); $\mathrm{N}_{\mathrm{t}}=$ nitrogênio total (total nitrogen). ${ }^{1}$ Paixão et al. (2012); **não determinado (not determined). 
Tabela 2. Coeficientes, estimadores e níveis de significância dos contrastes entre o tratamento de substrato comercial e aqueles com diferentes níveis de inserção da moinha no substrato em substituição ao substrato comercial Bioplant (coefficients, estimates and significance levels between estimators of the conventional substrate treatment, and those with different levels of moinha on the commercial substrate Bioplant). Santa Teresa, IFES, 2015.

\begin{tabular}{|c|c|c|c|c|c|c|c|}
\hline \multirow{4}{*}{ Variáveis de crescimento } & \multirow{3}{*}{$\begin{array}{c}\text { Substrato } \\
\text { comercial } \\
4\end{array}$} & \multicolumn{4}{|c|}{ Níveis de moinha do substrato (\%) } & \multirow{4}{*}{$\begin{array}{c}\text { Estimador } \\
-\end{array}$} & \multirow{4}{*}{$\begin{array}{c}\text { Erro } \alpha \\
-\end{array}$} \\
\hline & & 15 & 30 & 45 & 60 & & \\
\hline & & -1 & -1 & -1 & -1 & & \\
\hline & \multicolumn{5}{|c|}{ Média dos tratamentos } & & \\
\hline Altura de plantas $(\mathrm{cm})$ & 8,80 & 16,22 & 14,14 & 14,64 & 9,70 & $-19,50$ & 0,0000 \\
\hline Diâmetro do coleto (mm) & 1,15 & 2,00 & 1,92 & 2,09 & 1,21 & $-2,62$ & 0,0000 \\
\hline Número de folhas (un) & 4,61 & 5,61 & 5,69 & 5,71 & 5,15 & $-3,72$ & 0,0000 \\
\hline Matéria fresca de parte aérea (mg) & 491,02 & 1708,15 & 1522,49 & 1767,05 & 973,51 & $-4007,13$ & 0,0000 \\
\hline Matéria fresca de raiz (mg) & 112,03 & 452,87 & 370,77 & 425,94 & 277,11 & $-1078,57$ & 0,0277 \\
\hline Matéria seca de parte aérea (mg) & 49,66 & 132,02 & 95,30 & 124,17 & 55,70 & $-208,53$ & 0,0002 \\
\hline Ln da matéria seca de raiz (mg) & 2,00 & 3,18 & 2,98 & 3,12 & 2,39 & $-3,67$ & 0,0000 \\
\hline Matéria seca total (mg) & 57,56 & 156,97 & 116,49 & 147,05 & 69,88 & $-260,15$ & 0,0000 \\
\hline
\end{tabular}

aos 28 DAS.

A proporção estimada de 29,21\% de moinha proporcionou o maior valor de diâmetro do coleto $(2,11 \mathrm{~mm})$, enquanto o menor valor, $1,27 \mathrm{~mm}$, foi obtido no tratamento contendo $60 \%$ de moinha (Figura 1B). O maior diâmetro de coleto pode ser demonstrativo de plantas mais vigorosas (Santos et al., 2010). Em olerícolas, o diâmetro de coleto apresenta-se como umas das características fundamentais na hora de decidir se a muda está no momento do plantio.

A proporção estimada de $32,14 \%$ de moinha na composição do substrato apresentou o maior número de folhas por planta $(5,79)$, enquanto o menor valor $(5,25)$ foi obtido no tratamento contendo $60 \%$ de moinha (Figura 1C). Mesmo assim, tal valor foi superior àqueles encontrados por Ferreira et al. (2014) que obtiveram o melhor resultado $(3,84$ folhas por planta), aos 25 DAS, com o uso de um vermicomposto à base de esterco bovino $(80 \%)$ e esterco de pequenos ruminantes $(20 \%)$, na produção de mudas de tomate. Foi superior também aos resultados obtidos por Diniz et al. (2006) que encontraram valores variando entre 3,12 e 3,77 em substratos contendo húmus com níveis crescentes de vermiculita, na produção de mudas de tomate.

Verifica-se que os melhores resultados obtidos para as matérias frescas da parte aérea $(1.746,25 \mathrm{mg})$ e da raiz
(452,48 mg) foram obtidos nos substratos contendo $27,84 \%$ (Figura 1D) e 15\% (Figura 1E) de moinha, respectivamente. Já os menores valores foram obtidos nos substratos contendo $60 \%$ de moinha.

De acordo com as Figuras 1F, $1 \mathrm{G}$ e $1 \mathrm{H}$, o valor de $15 \%$ de moinha proporcionou as maiores quantidades de matéria seca de parte aérea $(131,8 \mathrm{mg})$, de raiz (24,84 mg, valor estimado de 3,21 mg para o Ln da MSR, Figura 1G) e total da planta $(157,21 \mathrm{mg})$, respectivamente, dentre os tratamentos aplicados (Figura $1 \mathrm{G})$. De maneira semelhante às outras variáveis, os menores valores médios foram obtidos nos substratos contendo $60 \%$ de moinha.

De um modo geral, verifica-se que os maiores valores obtidos das variáveis analisadas foram aqueles nos quais se utilizaram em torno de 15 a $32 \%$ de moinha na composição dos substratos. Com o aumento desta proporção, todas as variáveis apresentaram valores decrescentes. Acredita-se que tal fato possa estar associado à maior salinidade no substrato, evidenciada pela maior condutividade elétrica existente nesse resíduo (Tabela 1). A alta concentração de sais é um fator de estresse para as plantas, pois reduz o potencial osmótico e proporciona a ação dos íons sobre o protoplasma (Harter et al., 2014). A água é osmoticamente retida na solução salina, tornando-a cada vez menos disponível para as plantas.
O uso em torno de 15 a $32 \%$ de moinha na composição do substrato promoveu o melhor desenvolvimento das mudas do tomateiro como um todo, provavelmente por nessa faixa o efeito da salinidade não ter sobrepujado o efeito benéfico dos nutrientes, especialmente da maior concentração de nitrogênio da moinha (Tabela 1). Costa et al. (2013) obtiveram bons resultados nas variáveis de crescimento de plantas de tomate, especialmente na matéria seca da parte aérea, atribuindo tais resultados à liberação de nutrientes do material orgânico presente no substrato, ao longo dos 30 dias em que as mudas de tomate permaneceram nas bandejas sob boas condições de umidade.

Dentre os tratamentos aplicados, o substrato alternativo contendo de 15 a $32 \%$ de moinha $+15 \%$ fibra de coco + $5 \%$ casca de ovo $+48 \%$ a $65 \%$ de substrato comercial proporciona os maiores valores de altura, diâmetro de caule, número de folhas, matéria fresca e seca de raiz, matéria fresca e seca de parte aérea e matéria seca total.

A utilização da moinha numa proporção acima de $32 \%$ na composição do substrato para mudas do tomateiro promove o decréscimo dos valores de todas as variáveis analisadas, porém, todas as proporções de moinha no substrato estudadas promovem valores das variáveis de crescimento superiores àqueles obtidos quando se usa somente $o$ 


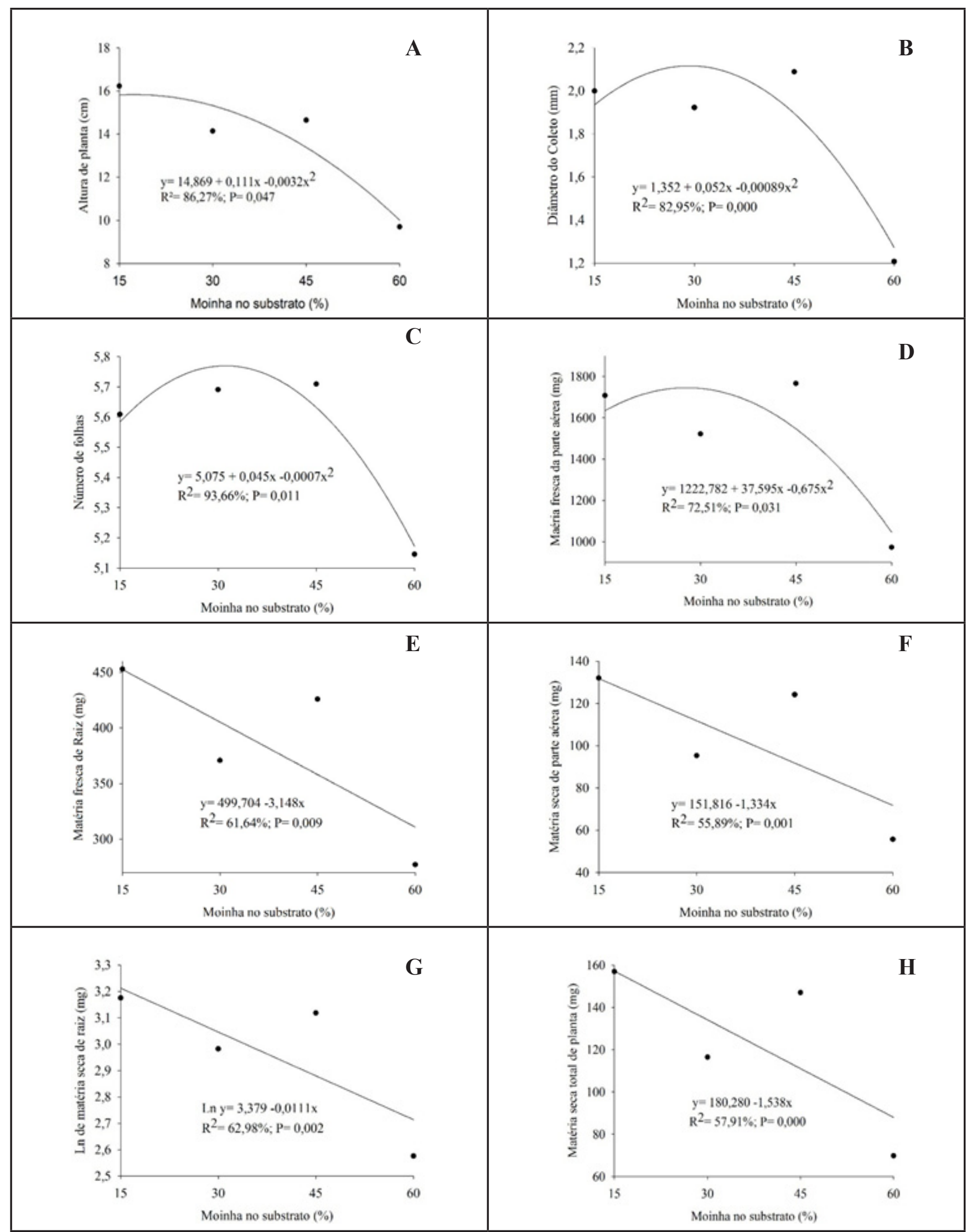

Figura 1. Altura de plantas (plant height) (A), diâmetro de coleto (diameter of the seedling base) (B), número de folhas (number of leaves) (C), matéria fresca de parte aérea (fresh matter of the aboveground part) (D), matéria fresca de raiz (fresh matter of root) (E), matéria seca de parte aérea (dry matter of the aboveground part) (F), matéria seca de raiz (dry matter of root) $(\mathrm{G})$ e matéria seca total (dry matter of the whole plant) $(\mathrm{H})$, em função de substratos com diferentes porcentagens de moinha em substituição ao substrato comercial Bioplant (depending on substrates with moinha levels replacing the commercial substrate Bioplant). Santa Teresa, IFES, 2015. 
substrato comercial. O uso de 5\% casca de ovo, $15 \%$ de fibra de coco e de 15 a $32 \%$ de moinha, em substituição ao substrato comercial, é uma alternativa de substrato para produção de mudas de tomate, permitindo um adequado destino a esses resíduos agrícolas.

\section{REFERÊNCIAS}

BARRETO, CVG; TESTEZLAF, R. 2014. Particle size distribution effects on physical characteristics of coconut and pine bark substrates. Revista de Ciências Agroveterinárias 13: 327-336.

CALDEIRA, MVW; GONÇALVES, EO; TRAZZI, PA; DELARMELINA, WM; ROCHA, RL. 2014. Crescimento de mudas de Eucalyptus grandis utilizando lodo de esgoto, fibra de coco e palha de café in natura. Floresta 44: 195.

CERQUEIRA, FB; FREITAS, GA; MACIEL, CJ; CARNEIRO, JSS; LEITE, RC. 2015. Produção de mudas de tomate cv. Santa Cruz em diferentes substratos. Journal of Bioenergy and Food Science 2: 39-45.

COSTA, CA; RAMOS, SJ; SAMPAIO, RA; GUILHERME, DO; FERNANDES, LA. 2007. Fibra de coco e resíduo de algodão para substrato de mudas de tomateiro. Horticultura Brasileira 25: 387-391.

COSTA, E; SANTO, TLE; SILVA, AP; SILVA, LE; OLIVEIRA, LC; BENETT, CGS; BENETT, KSS. 2015. Ambientes e substratos na formação de mudas e produção de frutos de cultivares de tomate cereja. Horticultura Brasileira 33: 110-118.
COSTA, LAM; COSTA, MSSM; PEREIRA, DC; BERNARDI, FH; MACCARI, S. 2013. Avaliação de substratos para a produção de mudas de tomate e pepino. Revista Ceres 60: 675-682.

DIAS, NS; PALÁCIO, VS; MOURA, KC; SOUSA NETO, ON. 2015. Crescimento do meloeiro em substrato de fibra de coco com solução nutritiva salina. Irriga 20: 1-12.

DINIZ, KA; GUIMARAES, STMR; LUZ, JMQ. 2006. Húmus como substrato para a produção de mudas de tomate, pimentão e alface. Bioscience Journal 22: 63-70.

FERREIRA, LL; ALMEIDA, AES; COSTA, LR; MEDEIROS, JF; PORTO, VCN. 2014. Vermicompostos como substrato na produção de mudas de tomate (Lycopersicon esculentum) e couve-folha (Brassica oleracea var. acephala). Revista Verde de Agroecologia e Desenvolvimento Sustentável 9: 256-263.

HARTER, LSH; HARTER, FS; DEUNER, D; MENEGHELLO, GE; VILLELA, FA. 2014. Salinidade e desempenho fisiológico de sementes e plântulas de mogango. Horticultura Brasileira 32: 80-85.

MAÇÃS, JES. 2008. Nitrogênio nítrico e amoniacal no desenvolvimento da parte aérea de milho cultivado em argissolo. Porto Alegre: UFRGS (Dissertação mestrado).

MATOS, AT. 2015. Manual de análise de resíduos sólidos e águas residuárias. Viçosa: UFV. $149 \mathrm{p}$.

MESQUITA, EF; CHAVES, LHG; FREITAS, BV; SILVA, GA; SOUSA, MVR; ANDRADE, R. 2012. Produção de mudas de mamoeiro em função de substratos contendo esterco bovino e volumes de recipientes. Revista Brasileira de Ciências Agrárias 7: 58-65.

MENEGHELLI, CM; MONACO, PAVL; HADDADE, IR; MENEGHELLI, LAM;
KRAUSE, MR. 2016. Resíduo da secagem dos grãos de café como substrato alternativo em mudas de café Conilon. Coffee Science 11: 330-335.

PAIXÃO, MVS; SCHMILDT, ER; MATTIELLO, HN; FERREGUETTI, GA; ALEXANDRE, RB. 2012. Frações orgânicas e mineral na produção de mudas de mamoeiro. Revista Brasileira de Fruticultura 34: 1105-1112.

RODRIGUES, ET; LEAL, PAM; COSTA, E; PAULA, TS; GOMES, VA. 2010. Produção de mudas de tomateiro em diferentes substratos e recipientes em ambiente protegido. Horticultura Brasileira 28: 483-488.

SANTOS, MR; SEDIYAMA, MAN; SALGADO, LT; VIDIGAL, SM; REIGADO, FR. 2010. Produção de mudas de pimentão em substratos à base de vermicomposto. Bioscience Journal 26: $572-578$.

SILVA JÚNIOR, JAS; GHEYI, HR; DIAS, NS; ARAÚJO, DA; GUEDES FILHO, DH. 2014. Substratos e diferentes concentrações da solução nutritiva preparada em água residuária no crescimento do girassol. Revista Ciência Agronômica 45: 696-707.

STEFFEN, GPK; ANTONIOLLI, ZI; STEFFEN, RB; MACHADO, RG. 2010. Casca de arroz e esterco bovino como substratos para a multiplicação de minhocas e produção de mudas de tomate e alface. Acta Zoológica Mexicana 26: 333-34.

SAEG. 2007. Sistema de Análises Estatísticas, Versão 9.1: Viçosa: UFV.

TAIZ, L; ZEIGER, E. 2013. Fisiologia vegetal. Porto Alegre: Artmed. 5.ed, p.820.

ZACCHEO, PVC; AGUIAR, RS; STENZEL, NMC; NEVES, CSVJ. 2013. Tamanho de recipientes e tempo de formação de mudas no desenvolvimento e produção de maracujazeiro-amarelo. Revista Brasileira de Fruticultura 35: 603-607. 